\title{
The Impact of Mild Stroke on Participation in Physical Fitness Activities
}

\author{
Mary Hildebrand, ${ }^{1,2}$ Megan Brewer, ${ }^{3}$ and Timothy Wolf ${ }^{2}$ \\ ${ }^{1}$ Department of Occupational Therapy, East Carolina University, HSB 3305K, Greenville, NC 27858, USA \\ ${ }^{2}$ Program in Occupational Therapy, Washington University School of Medicine in St. Louis, Saint Louis, MO 63108, USA \\ ${ }^{3}$ Memorial Medical Center, Springfield, IL 62781, USA
}

Correspondence should be addressed to Mary Hildebrand, hildebrandm@ecu.edu

Received 14 June 2011; Accepted 15 August 2011

Academic Editor: Julie A. Bernhardt

Copyright ( $) 2012$ Mary Hildebrand et al. This is an open access article distributed under the Creative Commons Attribution License, which permits unrestricted use, distribution, and reproduction in any medium, provided the original work is properly cited.

Objective. To compare participation in moderate to high intensity physical activities in persons before and after a mild stroke. Methods. We used data from the Cognitive Rehabilitation and Research Group to examine changes in moderate to high intensity physical activity participation in persons who had a mild stroke as defined by an NIH Stroke Scale score of less than $6(N=127)$. Using the Activity Card Sort, we compared the participants' high-demand leisure activity (leisure activities that are moderate to high intensity physical activities) participation at 6-months after stroke with their prestroke level. Results. We found a significant decrease in numbers of high-demand leisure activities in all participants and in each demographic group after mild stroke. Conclusion. These results suggest that persons after mild stroke are not retaining the high-demand leisure activities they were doing prior to their stroke. Health professionals must promote participation in high-demand leisure activities in patients with mild stroke as a tool to enhance health and fitness.

\section{Introduction}

Stroke incidence in the USA is estimated to be 795,000 per year with first-time attacks accounting for 610,000 and recurrent attacks accounting for 185,000 [1]. Cardiovascular comorbidities are common among stroke survivors with hypertension reported in $50-84 \%$ and heart disease in up to $75 \%$ of cases $[2,3]$. As a result, recurrent stroke and cardiac disease are the leading causes of mortality in stroke survivors [3-5].

Physical inactivity is a modifiable risk factor that can affect cardiovascular disease and stroke severity, incidence, and functional recovery $[2,5-8]$. Persons who were more physically active before stroke had less severe strokes and better functional outcomes than those who had been less active $[5,9,10]$. Participation in physical activity has been shown to improve performance of activities of daily living (ADL) and instrumental activities of daily living (IADL), improve fitness, and enhance perceived quality of life in stroke survivors $[11,12]$. In spite of these benefits, stroke survivors have the highest percentage of inactivity when compared to persons with other chronic conditions [13].

Physical activity has been defined as any bodily movement produced by skeletal muscle that results in energy expenditure varying from low, moderate, or high intensity $[6,14]$. Physical activity is positively correlated with physical fitness and is experienced in leisure time (including exercise as a subset of leisure), transportation, occupational, and household activities $[6,14]$.

The American Heart Association (AHA) has published specific physical activity guidelines for persons after stroke to address inactivity. They recommend 20-60 minutes of continuous or accumulated moderate to vigorous physical activity on three to seven days per week and strength, flexibility, and balance/coordination activities two to three days per week [12]. To meet these guidelines, it is essential that stroke survivors receive additional support to participate in physical activity. However, Tang and colleagues found that despite the common etiology between stroke and heart disease, physical activity and cardiac rehabilitation guidelines are 
generally not prescribed for the stroke population [15]. Schmid et al. reported that in a sample of Veteran's Affairs (VA) occupational and physical therapists, less than half of those surveyed work with their patients to enhance healthpromoting behaviors to prevent recurrent stroke in spite of published VA guidelines $[16,17]$.

Arguably stroke presents barriers that may prevent survivors from engaging in physical activity such as physical disability, depression, fatigue, lack of social reintegration, diminished motivation, and deconditioning [9]. However, the most prevalent form of stroke is mild stroke, defined by a National Institute of Health Stroke Scale (NIHSS) score less than $6[2,7]$. Wolf et al. found that $49.4 \%$ of stroke survivors had a mild stroke in their sample of 7,740 persons who had experienced stroke [18]. This confirmed the findings of Tellier and Rochette in their review of literature that found that mild stroke is more prevalent than moderate and severe strokes [4]. Studies have tended to use differing definitions of mild stroke, but generally the criteria have included having a maximum score on a prognostic or stroke scale, few disabling after-effects such as an absence of or only slight motor impairments, no significant aphasia or unilateral spatial neglect, no impairment in ability to perform activities of daily living (ADLs), enough mobility to get in and out of bed, or the ability to perform a toilet transfer $[2,7]$. Thus, we posit that persons who experience a mild stroke should be physically capable of following current poststroke guidelines and recommendations for participation in physical activities.

Since almost half of strokes are mild, it is important to examine the pre- and poststroke levels of moderate to high intensity physical activity participation in this population. This study has two objectives: (1) to determine if persons who had experienced a mild stroke retained their participation in prestroke moderate to high intensity leisure activities and (2) to determine if there are demographic differences in poststroke participation in moderate to high intensity leisure activities after mild stroke.

\section{Methods}

2.1. Subjects. This study is a secondary analysis of data collected for the Cognitive Rehabilitation Research Group (CRRG) at the Washington University School of Medicine in St. Louis. The CRRG recruits and registers patients from the Acute Neurology Stroke Service of Barnes-Jewish Hospital. The Washington University School of Medicine Human Research Protection Office (HRPO) reviewed and approved this study. Patients gave informed consent to be tested, to have their data placed in a registry, and to permit contact for future follow-up assessments. Data collected in the acute care setting included patients' demographic information, National Institute of Health Stroke Scale (NIHSS) score, and discharge dispensation information (i.e., if the patient was discharged to "no therapy," "home health," "outpatient," or "inpatient rehabilitation"). Between April 2003 and July 2007, the participants in the CRRG data registry were called and recruited 5-8 months (mean of 5.96 months) after stroke for a voluntary follow-up visit to administer the Activity
Card Sort (ACS) and other functional and performance measures. As stated above, for the purposes of this study, only the participants who had experienced a mild stroke as evidenced by a NIHSS score of less than 6 (i.e., scores from 0 to 5$)$ and who completed the ACS $(N=127)$ were included in this analysis.

2.2. Measures. The NIHSS is a measure that assesses neurologic impairment after stroke and is typically used as an indicator of stroke severity [19]. It is a 36-point scale that produces scores that range from 0 (no deficit indicated) to 42 (severe neurologic deficit). For this study, only participants with scores of less than 6 were included to indicate mild neurological deficits. A score of less than 6 on the NIHSS has also been found to strongly predict the likelihood of being discharged to home [20]. This population should theoretically have the physical capabilities to participate in physical activity. Several researchers have used the NIHSS score of less than 6 to differentiate between mild stroke to moderate and more severe strokes $[18,21,22]$.

The Activity Card Sort (ACS) is a standardized instrument that assesses participation in 80 instrumental, social, and low-demand and high-demand leisure activities [23]. It uses a Q-sort methodology or rank order procedure to obtain a general history of adults' participation in the four categories (instrumental, social, low-demand leisure, and high-demand leisure activities.) The ACS has been shown to have test-retest reliability of 0.897 [23] and the Israeli version has been shown to have internal consistency [24]. Content and construct validity have also been reported $[24,25]$. For the purpose of this study, only the high-demand leisure (HDL) category was examined because it includes 17 moderate to high intensity physical activities, for example, walking, running, swimming, bicycling, and gardening. For each of the activities, participants are asked to rate their level of participation using the following categories: "never done," "continue to do since the stroke," "given up since the stroke," "doing less often since the stroke," and "started to do this after the stroke." Standardized directions for scoring the ACS are as follows [23]. The total prestroke HDL activity score is a sum of activities that the participant rated as "continue to do since the stroke" plus "given up since the stroke" plus "doing less often since the stroke." The total poststroke HDL score is a sum of activities that the participant rated as "continue to do since the stroke" plus "started to do after the stroke" plus $1 / 2$ the number of activities rated "doing less often since the stroke." A score to describe the percentage of HDL activities retained and/or gained after stroke was calculated by dividing the total poststroke HDL activities score by the total prestroke HDL activities score. Thus, a higher score indicates better retention of prestroke HDL activity participation or a gain in participation in new HDL activities since the stroke.

2.3. Data Processing and Analysis. Data was analyzed using SPSS 16.0 (SPSS Inc., 2007) with a significance level of .05. Descriptive statistics were used to describe patient demographic characteristics, level of rehabilitation services, HDL activities before and after stroke, and the percentage of HDL 


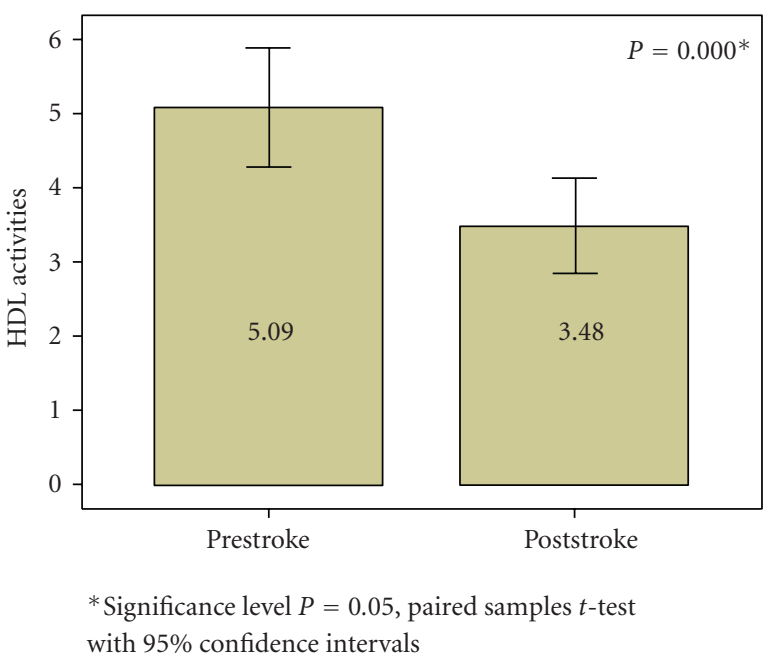

FIGURE 1: Mean number of HDL activities pre- and post-mild stroke for all participants $(N=127)$.

activities participants retained from pre-stroke to poststroke. We performed paired samples $t$-tests to determine if there was a significant difference when comparing the mean numbers of HDL activities before stroke with the mean numbers of HDL activities after stroke for all participants, each age group, gender, race, education level, and rehabilitation level. Independent samples $t$-tests compared mean numbers of HDL activities of the following pairs of groups: men and women, Caucasian and African American, and education of "high school or less" and "more than high-school." Rehabilitation services were combined into the following categories according to intensity level: no rehabilitation (discharged to home with no follow-up services), limited rehabilitation (outpatient rehabilitation, home health, or day treatment), or intensive rehabilitation (services in an inpatient rehabilitation hospital or a skilled nursing facility). A multiple regression analysis was conducted to evaluate how well age, education level, and rehabilitation group predicted poststroke HDL activity participation.

\section{Results}

Study participant demographic characteristics are presented in Table 1 . The mild stroke sample $(N=127)$ had a mean age of 63 with a wide range between 31 and 90 years. Because the HDL activity participation may differ widely by age, the participants were divided into three age groups based on the distribution of the sample for further analysis: 54 and younger, 55-69, and 70 and older. Our sample had more participants with a "high school education or less" and more Caucasians than African Americans, but was fairly evenly split by gender. The most frequently reported level of rehabilitation service was limited rehabilitation.

As shown in Figure 1, there was a significant decline in participation in HDL activities for all participants. Overall, they reported losing an average of more than 1.5 of their prestroke activities 6-8 months after experiencing a mild stroke. We found that there was a significant decline without regard to age, gender, race, education, or poststroke rehabilitation level (see Table 1). When examining the percentage of activities retained, we found that in all groups the percentage of prestroke activities that participants retained ranged from $53 \%$ to $76 \%$, that is, persons who have experienced a mild stroke are losing almost one-half to one-fourth of their prestroke HDL activities (see Table 1).

Men reported participating in significantly more HDL activities than women both before and after their stroke, but there was no significant difference in the percentage of activities that they retained (i.e., retaining approximately only 69 and $68 \%$, resp.) The same can be stated for those who had an education level beyond high school. They reported significantly more HDL activities than those with an education level of high school or less, but we found that there was no significant difference in the percentage of HDL activities retained between these groups (only 70 and 67\%, resp.) There were no significant differences in pre- or poststroke HDL activities reported by Caucasians or African Americans and no significant difference in percentage of HDL activities retained (only 72 and 64\%, resp.) (See Table 2).

Table 3 shows the results of the multiple regression analysis evaluating how well age, education level, and rehabilitation group predicted poststroke HDL activity participation. The linear combination of these factors was significantly related to poststroke HDL activity participation $\left(R^{2}=0.15\right.$, $P<0.000)$; however, age was the only significant predictor $(P<0.001)$.

\section{Discussion}

Participation in HDL activities declined significantly across all groups examined after mild stroke. Men and those who had an education level greater than a high school diploma reported significantly greater numbers of prestroke and poststroke HDL activities, but all groups reported an equivalent decline in HDL activity levels after mild stroke. We found no significant group differences in the decline from prestroke to poststroke when we compared men to women, Caucasians to African Americans, and between two levels of education. We also found that rehabilitation level received after stroke was not a significant predictor of HDL activity. As one would expect, we did find that age was a significant predictor of HDL activity participation after stroke [26].

Studies have reported that stroke survivors participate in less physical activity than others with chronic conditions [13]. However, although mild stroke makes up almost half of the occurrences of stroke, to our knowledge, there has been no description of the physical activity levels of participation before and after stroke and physical activity retention after stroke for this subpopulation. Since motor impairments are not characteristic of mild stroke as it is presently defined, we anticipated that there might not be significant poststroke declines from their prestroke HDL activity levels. In addition, we speculated that experiencing a mild stroke would be a "wake-up call" that might spur patients to seek opportunities to increase participation in prestroke HDL 
TABLe 1: Demographics, mean pre- and post-HDL activities, and mean \% HDL activities retained, $N=127$.

\begin{tabular}{|c|c|c|c|c|c|}
\hline & $N(\%)$ & $\begin{array}{c}\text { Mean HDL act. } \\
\text { prestroke }\end{array}$ & $\begin{array}{c}\text { Mean HDL act. } \\
\text { poststroke }\end{array}$ & $P$ value & $\begin{array}{c}\text { HDL act. \% } \\
\text { retained (post/pre) }\end{array}$ \\
\hline NIHSS score: mean (SD), range & 2.39 (1.54), $0-5$ & & & & \\
\hline Age, years: mean (SD), range & 62.55 (13.51), 31-90 & & & & \\
\hline 54 years or younger & $38(30)$ & 7.47 & 5.18 & $0.000^{*}$ & $69 \%$ \\
\hline 55 to 69 years & $48(38)$ & 4.75 & 3.47 & $0.000^{*}$ & $73 \%$ \\
\hline 70 years or older & $41(32)$ & 3.27 & 1.91 & $0.000^{*}$ & $58 \%$ \\
\hline \multicolumn{6}{|l|}{ Gender } \\
\hline Female & $65(51)$ & 3.68 & 2.52 & $0.000^{*}$ & $68 \%$ \\
\hline Male & $62(49)$ & 6.56 & 4.48 & $0.000^{*}$ & $69 \%$ \\
\hline \multicolumn{6}{|l|}{ Race } \\
\hline Caucasian & $74(58)$ & 5.18 & 3.78 & $0.000^{*}$ & $72 \%$ \\
\hline African American & $53(42)$ & 4.96 & 3.06 & $0.000^{*}$ & $64 \%$ \\
\hline $\begin{array}{l}\text { Education: mean no. of years (SD), } \\
\text { range }\end{array}$ & $12.87(2.88), 6-22$ & & & & \\
\hline High school or less & $68(54)$ & 3.96 & 2.68 & $0.000^{*}$ & $67 \%$ \\
\hline More than high school & $59(46)$ & 6.39 & 4.41 & $0.000^{*}$ & $70 \%$ \\
\hline \multicolumn{6}{|l|}{ Rehabilitation services poststroke } \\
\hline None & $29(23)$ & 5.79 & 4.03 & $0.000^{*}$ & $70 \%$ \\
\hline Limited & $60(47)$ & 5.08 & 3.84 & $0.000^{*}$ & $76 \%$ \\
\hline Intensive & $33(26)$ & 4.30 & 2.27 & $0.000^{*}$ & $53 \%$ \\
\hline Missing data & $5(4)$ & & & & \\
\hline
\end{tabular}

* Differences between mean pre- and post-mild stroke HDL activity levels significant at $P<0.004$ after Bonferroni correction.

TABLE 2: Comparison of mean pre- and post-mild stroke HDL activities and $\%$ retained by gender, race, and education, $N=127$.

\begin{tabular}{|c|c|c|c|c|c|c|c|}
\hline & $n$ & $\begin{array}{l}\text { Mean HDL act. } \\
\text { prestroke }\end{array}$ & $P$ value & $\begin{array}{l}\text { Mean HDL act. } \\
\text { poststroke }\end{array}$ & $P$ value & $\begin{array}{c}\text { Mean HDL activities } \\
\% \text { retained }\end{array}$ & $P$ value \\
\hline \multicolumn{8}{|l|}{ Gender } \\
\hline Female & 65 & 3.68 & \multirow{2}{*}{$0.000^{*}$} & 2.52 & \multirow{2}{*}{$0.002^{*}$} & 67.82 & \multirow{2}{*}{0.875} \\
\hline Male & 62 & 6.56 & & 4.48 & & 68.83 & \\
\hline \multicolumn{8}{|l|}{ Race } \\
\hline Caucasian & 74 & 5.18 & \multirow{2}{*}{0.797} & 3.78 & \multirow{2}{*}{0.799} & 71.57 & \multirow{2}{*}{0.227} \\
\hline African-American & 53 & 4.96 & & 3.06 & & 63.73 & \\
\hline \multicolumn{8}{|l|}{ Education } \\
\hline High school or less & 68 & 3.96 & \multirow{2}{*}{$0.003^{*}$} & 2.68 & \multirow{2}{*}{$0.003^{*}$} & 67.18 & \multirow{2}{*}{0.410} \\
\hline More than high school & 59 & 6.39 & & 4.41 & & 69.54 & \\
\hline
\end{tabular}

* Differences between mean HDL activity levels significant at $P<0.005$ after Bonferroni correction.

TABle 3: Regression results for poststroke HDL activity participation.

\begin{tabular}{lcc}
\hline & Beta & Standard error \\
\hline Constant & 7.701 & 2.423 \\
Age & $-0.081^{*}$ & 0.024 \\
Education & 0.166 & 0.113 \\
Rehabilitation Groups & -0.652 & 0.443 \\
\hline
\end{tabular}

$R^{2}=0.153 ;{ }^{*} P<0.001$.

activities or participate in new ones, especially given that our stroke rehabilitation guidelines include patient education on the importance of increasing physical activity to maintain health and improve possible cardiovascular comorbidities. However, our findings indicate that persons after mild stroke decreased participation in a significant number of their prestroke HDL activities and did not initiate new HDL activities as a means to increase physical activity in an effort to meet poststroke guidelines [2, 7].

While HDL activity numbers dropped despite the need for increased participation, we acknowledge that there are several barriers associated with the lack of physical activity after stroke seemingly unrelated to motor impairment. These include depression, fatigue, social reintegration problems after hospitalization, decreased motivation, deconditioning, 
other acute illness, and lack of familial support [12]. We contend that many of these barriers can be addressed by rehabilitation professionals and urge them to go beyond the typical focus on activities of daily living with their patients with mild stroke [27].

Our results support what other studies have found; women, persons with less education, and older adults report lower participation in moderate to high intensity physical activities than men, persons with higher education, and younger adults [28]. Although all groups should be targeted for intervention to retain and increase their moderate to high intensity physical activity, medical professionals in rehabilitation should be acutely aware of providing appropriate recommendations and interventions to these higher risk groups.

Most stroke survivors have long-standing lifestyle risk factors such as poor diet and obesity, smoking, and inactivity [7]. Intervention guidelines for poststroke rehabilitation have been published that include recommendations for therapists to address healthy lifestyle behaviors in their patients after stroke $[7,12,17]$. Because they are less likely to have residual physical disabilities that make it difficult to participate in physical activity, persons who have experienced a mild stroke are the ideal candidates for self-management programs, behavior change education, and physical activity interventions in rehabilitation or community settings. Our results show that the level of rehabilitation services received after stroke was not a significant predictor of patients' participation in HDL activities. Participants in all levels showed a significant decline in numbers of HDL activities. It is vital that we become familiar with the AHA and VA guidelines and devote time and effort to these modifiable lifestyle risk factors with all stroke survivors $[7,12,17]$. To accomplish this in rehabilitation settings, better understanding of the HDL activity preferences of persons who have experienced a mild stroke has been recommended to help increase their participation [29]. Additionally, to increase participation in this group, we would also recommend that all patients with mild stroke be given an assessment, such as the ACS, to identify prestroke HDL activities and other physical activities that may be meaningful for the individual. Therapists could use these activities to motivate participation in therapy, increase self-efficacy in performing them, and find community resources to address perceived barriers to participation in them [30].

There are several possible limitations with our study. All subjects volunteered to participate in the follow-up assessment six months after their stroke and may not be a representative sample of persons with mild stroke. The level of rehabilitation received on discharge from the acute care hospital may be dependent on the patient's comorbidities, not on impairment caused by the mild stroke, thus, the comorbid condition may be the deciding factor affecting the patient's ability to participate in an HDL activity. In addition, others have argued that the NIHSS assessment may not measure the deficits that affect a person with mild stroke who experiences problems with participation in everyday activities [18]. The ACS is a self-report measure and may not accurately reflect actual participation in HDL activities before and after stroke. In addition, the ACS measures the numbers of HDL activities, but does not measure the intensity, frequency, or duration of each activity. For example, a person after stroke may have given up gardening, but perform more walking and, thus, would be scored as having decreased his/her number of HDL activities.

\section{Conclusions}

Participation in HDL activities declines in all groups even after a mild stroke with few identified neurological impairments. It is critical for rehabilitation specialists to address this decline in all persons who have experienced a mild stroke. By meeting recommended guidelines, persons with mild stroke may ameliorate common cardiovascular comorbidities, improve performance in daily activities, improve fitness, and enhance quality of life. The next step to increase participation in HDL activities in persons with mild stroke will be to design and test behavior change and self-management programs that will successfully increase healthy lifestyle behaviors in this population.

\section{Acknowledgments}

The authors acknowledge the James S. McDonnell Foundation (Grant 220020087, Carolyn Baum, PI) for the support of this study. In addition, they would like to thank the faculty, staff, and students in the Cognitive Rehabilitation Research Group at the Washington University in St. Louis School of Medicine.

\section{References}

[1] D. Lloyd-Jones, R. J. Adams, T. M. Brown et al., "Executive summary: heart disease and stroke statistics-2010 update: a report from the american heart association," Circulation, vol. 121, no. 7, pp. e46-e215, 2010.

[2] L. B. Goldstein, C. D. Bushnell, R. J. Adams et al., "Guidelines for the primary prevention of stroke: a guideline for healthcare professionals from the American Heart Association/American Stroke Association," Stroke, vol. 42, pp. 517-584, 2011.

[3] E. J. Roth, "Heart disease in patients with stroke: incidence, impact, and implications for rehabilitation part 1: classification and prevalence," Archives of Physical Medicine and Rehabilitation, vol. 74, no. 7, pp. 752-760, 1993.

[4] M. Tellier and A. Rochette, "Falling through the cracks: a literature review to understand the reality of mild stroke survivors," Topics in Stroke Rehabilitation, vol. 16, no. 6, pp. 454-462, 2009.

[5] D. Deplanque, I. Masse, C. Lefebvre, C. Libersa, D. Leys, and R. Bordet, "Prior TIA, lipid-lowering drug use, and physical activity decrease ischemic stroke severity," Neurology, vol. 67, no. 8, pp. 1403-1410, 2006.

[6] World Health Organization (n.d.), "Global strategy on diet, physical activity and health: physical activity," 2011, http://www.who.int/dietphysicalactivity/pa/en/index.html.

[7] K. L. Furie, S. E. Kasner, R. J. Adams et al., "Guidelines for the prevention of stroke in patients with stroke or transient ischemic attack: a guideline for healthcare professionals from 
the American Heart Association/American Stroke Association," Stroke, vol. 42, pp. 227-276, 2011.

[8] A. V. Chobanian, G. L. Bakris, H. R. Black et al., "The seventh report of the Joint National Committee on prevention, detection, evaluation, and treatment of high blood pressure: the JNC 7 report," Journal of the American Medical Association, vol. 289, no. 19, pp. 2560-2572, 2003.

[9] L. H. Krarup, T. Truelsen, C. Gluud et al., "Prestroke physical activity is associated with severity and long-term outcome from first-ever stroke," Neurology, vol. 71, no. 17, pp. 13131318, 2008.

[10] N. Stroud, T. M. L. Mazwi, L. D. Case et al., "Prestroke physical activity and early functional status after stroke," Journal of Neurology, Neurosurgery and Psychiatry, vol. 80, no. 9, pp. 1019-1022, 2009.

[11] H. Hoenig, N. Nusbaum, and K. Brummel-Smith, "Geriatric rehabilitation: state of the art," Journal of the American Geriatrics Society, vol. 45, no. 11, pp. 1371-1381, 1997.

[12] N. F. Gordon, M. Gulanick, F. Costa et al., "Physical activity and exercise recommendations for stroke survivors: an American Heart Association scientific statement from the Council on Clinical Cardiology, Subcommittee on Exercise, Cardiac Rehabilitation, and Prevention; the Council on Cardiovascular Nursing; the Council on Nutrition, Physical Activity, and Metabolism; and the Stroke Council," Stroke, vol. 35, no. 5, pp. 1230-1240, 2004.

[13] M. Y. C. Pang, J. J. Eng, A. S. Dawson, and S. Gylfadóttir, “The use of aerobic exercise training in improving aerobic capacity in individuals with stroke: a meta-analysis," Clinical Rehabilitation, vol. 20, no. 2, pp. 97-111, 2006.

[14] C. J. Caspersen, K. E. Powell, and G. Christenson, "Physical activity, exercise and physical fitness: definitions and distinctions for health-related research," Public Health Reports, vol. 100, no. 2, pp. 126-131, 1985.

[15] A. Tang, V. Closson, S. Marzolini, P. Oh, W. McIlroy, and D. Brooks, "Cardiac rehabilitation after stroke-need and opportunity," Journal of Cardiopulmonary Rehabilitation and Prevention, vol. 29, no. 2, pp. 97-104, 2009.

[16] A. A. Schmid, L. Butterbaugh, C. Egolf, V. Richards, and L. Williams, "Prevention of secondary stroke in VA: role of occupational therapists and physical therapists," Journal of Rehabilitation Research and Development, vol. 45, no. 7, pp. 1019-1026, 2008.

[17] B. Bates, J. Y. Choi, P. W. Duncan et al., "Veterans affairs/department of defense clinical practice guideline for the management of adult stroke rehabilitation care: executive summary," Stroke, vol. 36, no. 9, pp. 2049-2056, 2005.

[18] T. J. Wolf, C. Baum, and L. T. Connor, "Changing face of stroke: implications for occupational therapy practice," American Journal of Occupational Therapy, vol. 63, no. 5, pp. 621$625,2009$.

[19] T. Brott, H. P. Adams, C. P. Olinger et al., "Measurements of acute cerebral infarction: a clinical examination scale," Stroke, vol. 20, no. 7, pp. 864-870, 1989.

[20] D. Schlegel, S. J. Kolb, J. M. Luciano et al., "Utility of the NIH stroke scale as a predictor of hospital disposition," Stroke, vol. 34, no. 1, pp. 134-137, 2003.

[21] A. Rochette, N. K Korner-Bitensky, D. Bishop et al., "Study protocol of the YOU CALL-WE CALL TRIAL: impact of a multimodal support intervention after a "mild" stroke," BMC Neurology, vol. 10, article 3, 10 pages, 2010, http://www .biomedcentral.com/1471-2377/10/3.

[22] D. F. Edwards, M. Hahn, C. Baum, and A. W. Dromerick, "The impact of mild stroke on meaningful activity and life satisfaction," Journal of Stroke and Cerebrovascular Diseases, vol. 15, no. 4, pp. 151-157, 2006.

[23] C. M. Baum and D. F. Edwards, The Washington University Activity Card Sort, PenUltima Press, St Louis, Mo, USA, 2001.

[24] N. Katz, H. Karpin, A. Lak, T. Furnman, and A. HartmanMaeir, "Participation in occupational performance: reliability and validity of the activity card sort," Occupational Therapy Journal of Rehabilitation, vol. 23, no. 1, pp. 10-17, 2003.

[25] D. Sachs and N. Josman, "The activity card sort: a factor analysis," Occupational Therapy Journal of Rehabilitation, vol. 23, no. 4, pp. 165-174, 2003.

[26] M. C. Ashe, W. C. Miller, J. J. Eng, and L. Noreau, "Older adults, chronic disease and leisure-time physical activity," Gerontology, vol. 55, no. 1, pp. 64-72, 2009.

[27] L. G. Richards, N. K. Latham, D. U. Jette, L. Rosenberg, R. J. Smout, and G. DeJong, "Characterizing occupational therapy practice in stroke rehabilitation," Archives of Physical Medicine and Rehabilitation, vol. 86, no. 12, supplement 2, pp. S51-S60, 2005.

[28] W. L. Haskell, I. M. Lee, R. R. Pate et al., "Physical activity and public health: updated recommendation for adults from the American College of Sports Medicine and the American Heart Association," Circulation, vol. 116, no. 9, pp. 1081-1093, 2007.

[29] G. Banks, J. Bernhardt, L. Churilov, and T. B. Cumming, "Exercise preferences are different after stroke," Stroke Research and Treatment, vol. 2012, Article ID 890946, 9 pages, 2012.

[30] J. H. Rimmer, E. Wang, and D. Smith, "Barriers associated with exercise and community access for individuals with stroke," Journal of Rehabilitation Research and Development, vol. 45, no. 2, pp. 315-322, 2008. 


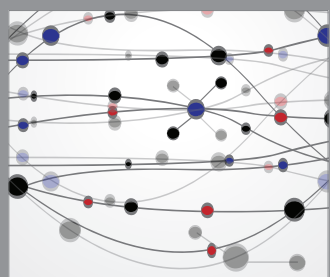

The Scientific World Journal
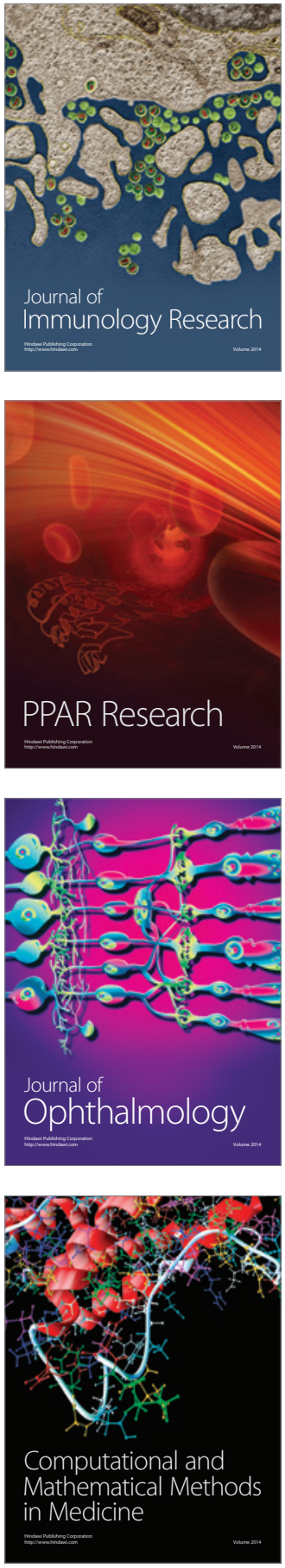

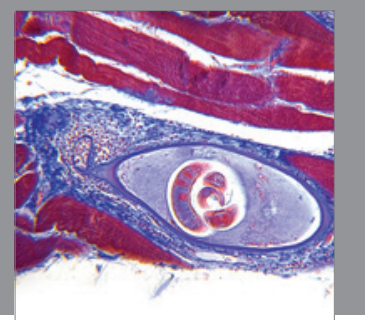

Gastroenterology

Research and Practice
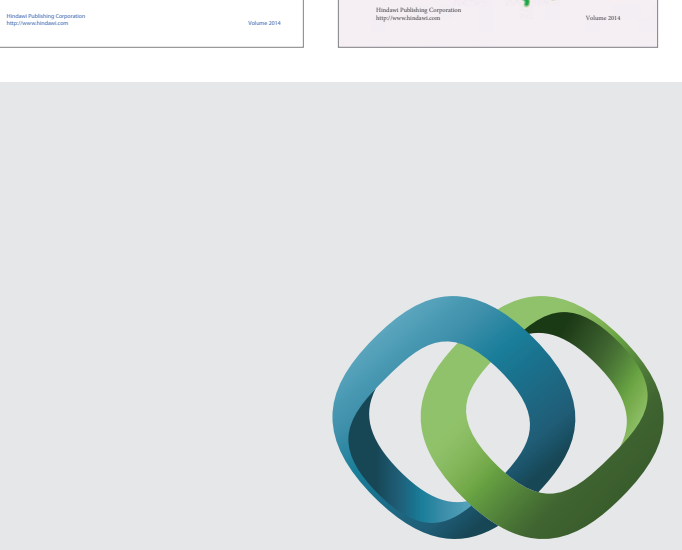

\section{Hindawi}

Submit your manuscripts at

http://www.hindawi.com
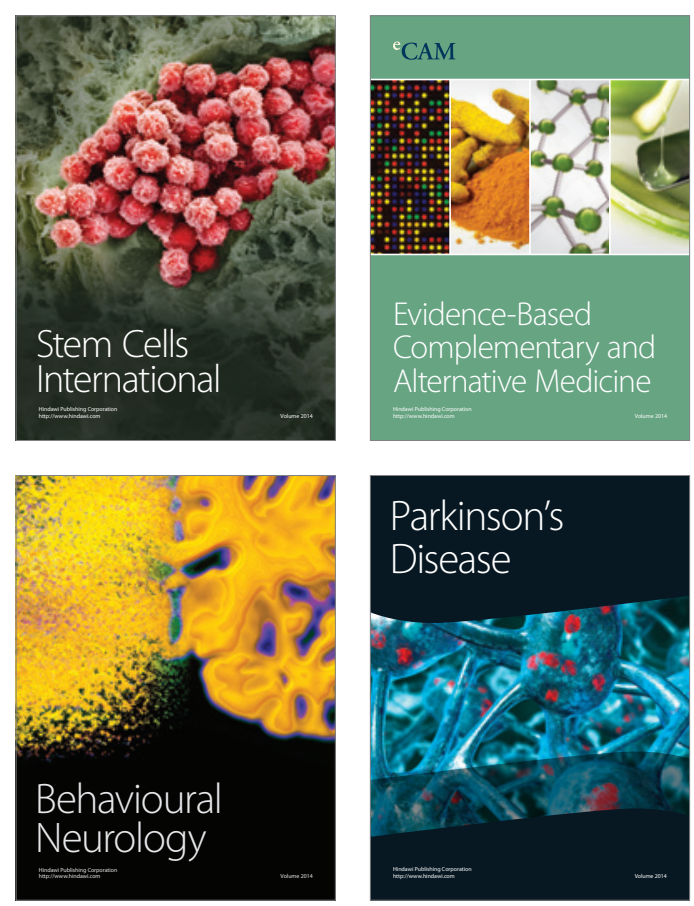

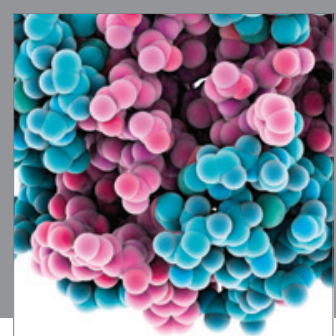

Journal of
Diabetes Research

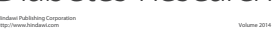

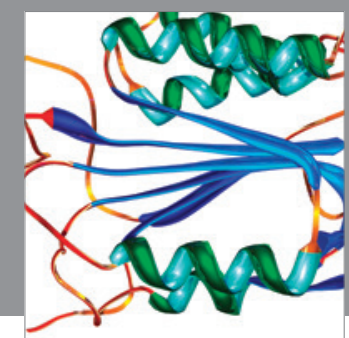

Disease Markers
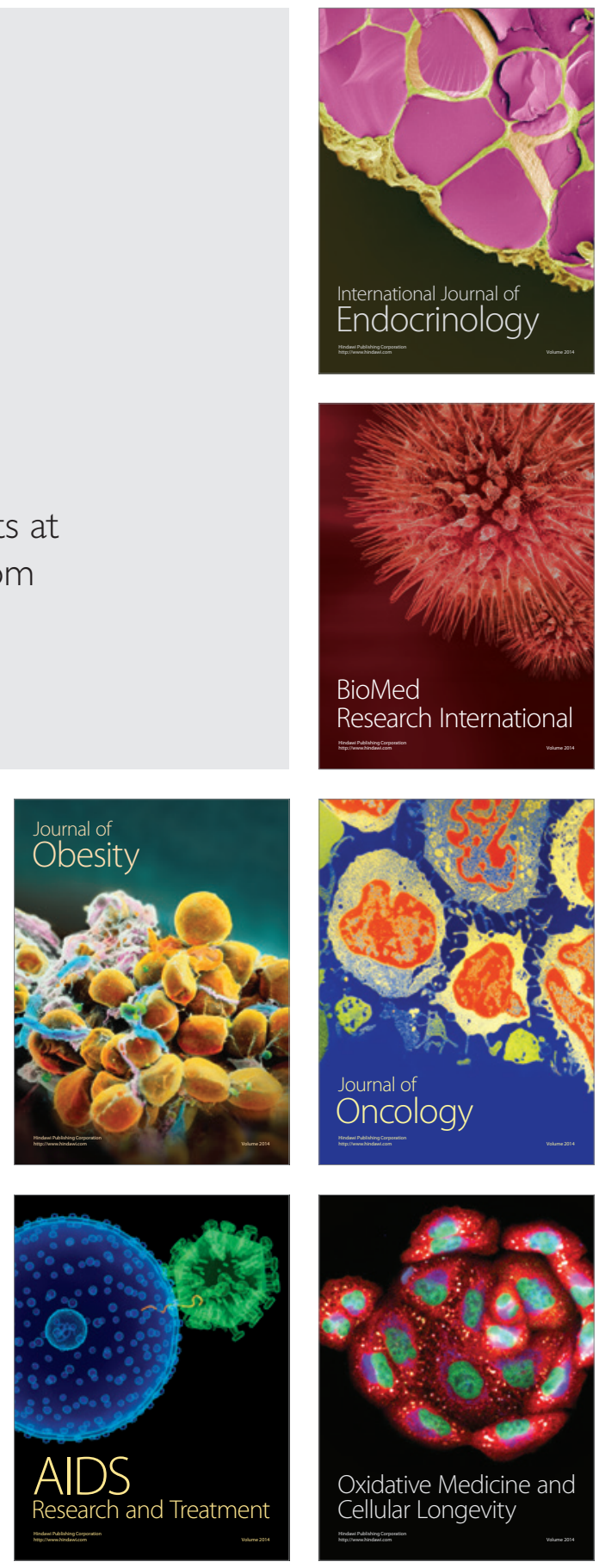